\title{
Results of Short-Term Follow-Up in BI-RADS 3 and $4 a$ Breast Lesions with a Histological Diagnosis of Fibroadenoma at Percutaneous Needle Biopsy
}

\author{
Paola Clauser $^{\mathrm{a}}$ Massimo Bazzocchi $^{\mathrm{b}}$ Magda Marcon $^{\mathrm{c}}$ Viviana Londero $^{\mathrm{b}}$ Chiara Zuiani $^{\mathrm{b}}$ \\ a Department of Biomedical Imaging and Image-Guided Therapy, Medical University of Vienna, Vienna, Austria; \\ bInstitute of Diagnostic Radiology, University of Udine, Udine, Italy; \\ ${ }^{\mathrm{c}}$ Institute of Diagnostic and Interventional Radiology, University Hospital Zurich, Zurich, Switzerland
}

\section{Keywords}

Breast · Fibroadenoma · Biopsy · Ultrasound · Mammography

\section{Summary}

Objective: To evaluate the usefulness of short-term (6 months) follow-up in patients with Breast Imaging Report and Data System (BI-RADS) 3 and 4a lesions, after a diagnosis of fibroadenoma at an image-guided biopsy. Patients and Methods: The data of 318 women with 349 biopsy-proven fibroadenomas, a 6-month follow-up, and a follow-up of $\geq 24$ months were retrospectively reviewed. Information on clinical history, lesion characteristics on ultrasound (US), mammography, and magnetic resonance imaging (MRI), BI-RADS classification, and follow-up was collected. The false-negative (FN) rate and the negative predictive value (NPV) for the biopsy were calculated. Results: 43 patients (13.5\%) presented with a palpable nodule; $18(5.7 \%)$ had a history of breast cancer. There were 334 lesions visible on US (95.7\%), 57 on US and mammography (16.3\%), and 15 on mammography only $(4.3 \%)$; 37 lesions were first detected on MRI. All lesions were stable at 6 months. After an at least 1-year follow-up, 4 lesions changed their features and were excised. Histology showed 1 invasive lobular cancer, 1 ductal carcinoma in situ, 1 phyllodes tumor, and 1 papilloma. The FN rate of the needle biopsy was $1.1 \%$ and the NPV was $98.9 \%$. Conclusion: For lesions initially described as BI-RADS 3 and 4 a with a histological diagnosis of fibroadenoma after biopsy, short-term follow-up can be avoided.

(C) 2017 S. Karger GmbH, Freiburg

\section{KARGER}

(๑) 2017 S. Karger GmbH, Freiburg

\section{Introduction}

The typical imaging features of fibroadenoma on mammography and ultrasound (US) are well known, and in the majority of cases, no further characterization (e.g., histologic/cytologic sampling, breast magnetic resonance imaging (MRI)) is required $[1,2]$. When a fibroadenoma-like lesion is detected for the first time, it is common practice to classify it as probably benign (BI-RADS 3 using the American College of Radiology (ACR) Breast Imaging Reporting and Data System (BI-RADS) [2]) and a short-term imaging follow-up of 6 months is recommended [3-5].

Nevertheless, some patient-related factors, such as anxiety, pregnancy, on-going hormonal therapy, and difficulties in guaranteeing a regular follow-up, may lead to the decision to immediately characterize the lesion, using fine-needle aspiration cytology or needle biopsy. Furthermore, a significant number of fibroadenomas may present with imaging features that can simulate more aggressive lesions, such as increased vascularity, lack of well-defined margins, or microcalcifications. The lesions are then classified as BI-RADS 4, and histological characterization is mandatory.

When a biopsy is performed, the risk of incomplete and/or incorrect sampling may lead to false-negative (FN) results, defined as a histological diagnosis of normal breast tissue or a benign lesion when a malignant or high-risk lesion is present [6]. On the basis of these observations, in our institution, a 6-month re-scan with US and/or mammography is routinely recommended after a biopsy with a histological diagnosis of fibroadenoma. When the lesion is stable at 6 and 12 months after biopsy, the woman returns to regular screening.

We hypothesized that, for lesions presenting with a low likelihood of malignancy, short-term follow-up after biopsy can be avoided. To confirm this hypothesis, we evaluated the follow-up of BI-RADS 3 and 4 a lesions with a histological diagnosis of fibroadenoma at image-guided needle biopsy. 


\section{Patients and Methods}

\section{Patient Population}

Lesions with a histological diagnosis of fibroadenoma after needle biopsy performed at our institution between January 2000 and December 2009 were retrospectively evaluated. Overall, 839 lesions in 781 women were found. Indications for the examination were: recall from the regional screening program, personal screening, symptoms, and second-look US examination for lesions detected on breast MRI.

Inclusion criteria were: (a) 14-gauge (G) US-guided core needle biopsy (CNB) or 11-G vacuum-assisted biopsy (VAB) under stereotactic guidance performed at our institution with a diagnosis of fibroadenoma; (b) occurrence of a short-term, 6-month follow-up with US alone or in combination with mammography, performed at our institution; and (c) an overall follow-up after biopsy of at least 24 months.

Exclusion criteria were: (a) incomplete follow-up (304 patients, 38.9\%; 85 patients had long-term follow-up but no control at 6 months, and 219 patients had an overall follow-up shorter than 24 months); (b) incomplete histological data (6 patients were operated at a different institute, $0.8 \%$ ); (c) absence of data on lesion characteristics and BI-RADS classifications and inability to retrieve the images (68 patients, 8.7\%); (d) lesion classified as BI-RADS $4 \mathrm{~b}, 4 \mathrm{c}$, or 5 (85 patients, $10.9 \%$ )

The final study population consisted of 318 women (mean age \pm standard deviation (SD), $49.1 \pm 9$ years; range, 17-81 years) with 349 biopsy-proven fibroadenomas.

\section{Data Collection}

Institutional review board approval was granted; due to the retrospective nature of the study, the patients' informed consent was waived. In all cases, informed consent was obtained for the interventional procedure.

Two radiologists, each with 3 years of experience in breast imaging, reviewed all the included cases The following information was recorded: (a) presence of symptoms at the initial evaluation (palpable nodule, nipple discharge); (b) risk factors for breast cancer (family or personal history of breast cancer); (c) presence of concomitant lesions, in the same or contralateral breast, with particular attention to malignant lesions; (d) lesion characteristics for each imaging modality; and (e) modifications of lesion features during follow-up.

\section{Ultrasound}

All women underwent breast US, performed by a radiologist dedicated to breast imaging. For each lesion, morphology, margins, echogenicity, posterior acoustic shadowing or enhancement, vascularization, and size were evaluated, as reported by Stavros et al. [7]. No lesions 'taller than wide' were found, so the descriptor was not considered in the analysis. CNB under US guidance was performed with a 14-G needle and 4-6 samples were collected.

\section{Mammography}

Mammography was performed in 203 patients, in both standard views (cranio-caudal and medio-lateral-oblique) in all cases. The presence of a mass and of calcifications and the size of the findings were evaluated. VAB under stereotactic guidance was performed in all lesions without a corresponding finding on US, using an 11-G needle; 10-12 samples were collected.

\section{Magnetic Resonance Imaging}

Breast MRI was performed in 57 women. Some of the lesions were identified at the MRI examination performed for breast cancer staging, during screening in high-risk patients, and during follow-up for previous breast cancer or for a problem-solving rationale. Studies were performed initially on a 1-T and then on a 1.5-T magnetic resonance (MR) scanner, with dedicated breast coils, after intravenous injection of gadolinium contrast agent. The study protocol included contrast-enhanced 3-dimensional (3D) gradient echo, T1weighted sequences acquired in a coronal (until 2010) or axial (from 2011) plane, and a short-tau inversion recovery (STIR) T2-weighted sequence acquired in the axial plane. No MR-guided biopsies with a diagnosis of fibroadenoma were obtained during the considered interval time.
Table 1. Ultrasound characteristics of the 349 lesions included in the study: cases with a conclusive diagnosis of fibroadenoma or malignant/borderline lesions (B3)

\begin{tabular}{|c|c|c|c|}
\hline & $\begin{array}{l}\text { All lesions, } \\
\text { n (\%) }\end{array}$ & $\begin{array}{l}\text { Fibroadenomas, } \\
\text { n (\%) }\end{array}$ & $\begin{array}{l}\text { Malignant } \\
\text { lesions/B3, n (\%) }\end{array}$ \\
\hline \multicolumn{4}{|l|}{ Shape } \\
\hline Oval & $232(69.5)$ & $230(69.7)$ & $2(50.0)$ \\
\hline Round & $43(12.9)$ & $42(12.7)$ & $1(25.0)$ \\
\hline Lobulated & $59(17.6)$ & $58(17.6)$ & $1(25.0)$ \\
\hline \multicolumn{4}{|l|}{ Margins } \\
\hline Well-defined & $260(77.8)$ & $257(77.9)$ & $3(75.0)$ \\
\hline Indistinct & $74(22.2)$ & $73(22.1)$ & $1(25.0)$ \\
\hline \multicolumn{4}{|l|}{ Shadowing } \\
\hline Present & $31(9.3)$ & $31(9.4)$ & $0(0)$ \\
\hline Absent & $303(90.7)$ & $299(90.6)$ & $4(100)$ \\
\hline \multicolumn{4}{|c|}{ Posterior enhancement } \\
\hline Present & $16(4.8)$ & $16(4.9)$ & $0(0)$ \\
\hline Absent & $318(95.2)$ & $314(95.1)$ & $4(100)$ \\
\hline \multicolumn{4}{|l|}{ Vascularization } \\
\hline Present & $73(21.8)$ & $72(21.8)$ & $1(25.0)$ \\
\hline Absent & $261(78.2)$ & $258(78.2)$ & $3(75.0)$ \\
\hline \multicolumn{4}{|l|}{ Dimensions } \\
\hline$\leq 10 \mathrm{~mm}$ & $174(52.1)$ & $173(52.4)$ & $1(25.0)$ \\
\hline $11-20 \mathrm{~mm}$ & $134(40.1)$ & $132(40.0)$ & $2(50.0)$ \\
\hline$\geq 21 \mathrm{~mm}$ & $26(7.8)$ & $25(7.6)$ & $1(25.0)$ \\
\hline
\end{tabular}

Statistical Analysis

For the statistical analysis, Microsoft Excel (Microsoft Corporation, Redmond, WA, USA) and the software MedCalc for Windows v. 9.1.0.1 (Ostend, Belgium) were used.

We defined as FN those cases with an initial histological diagnosis of fibroadenoma not confirmed by a later histological diagnosis during the followup. The negative predictive value (NPV) of image-guided biopsy with a diagnosis of fibroadenoma was calculated.

\section{Results}

Of the 318 women included, 47 women (14.8\%) were referred to our institution because of symptoms: 43 (13.5\%) for a palpable lesion and $4(1.2 \%)$ for nipple discharge.

Four women (1.2\%) had a personal history of breast cancer, and 14 women $(4.4 \%)$ had a family history of breast cancer, with at least a first-degree relative with the disease. In 17 women (5.3\%), a concomitant cancer was diagnosed: In 5 cases, the cancer was in the same breast, in 10 cases, in the contralateral breast, and in 2 cases, the cancer was bilateral.

The other 248 women $(78.0 \%)$ did not present any symptoms, nor any risk factor for breast cancer and/or other lesions.

Of the 349 lesions, 334 were visible on US (95.7\%) (table 1) and 15 were visible only on mammography (4.3\%). A mammographic correlate was found in 57 of the 334 US findings (16.3\%) (table 2). 41 lesions in 37 women were described first on breast MRI (11.7\%) and all were detected at second-look US (table 3).

According to the ACR BI-RADS lexicon, 313 lesions were classified as BI-RADS $3(90 \%)$ and 36 as BI-RADS $4 \mathrm{a}(10 \%)$. 
Table 2. Characteristics of the lesions visible on mammography

\begin{tabular}{lcc}
\hline Mammographic finding & $\begin{array}{l}\text { Lesions with a correlated } \\
\text { US finding }(\mathrm{N}=57), \mathrm{n}(\%)\end{array}$ & $\begin{array}{l}\text { Lesions without a correlated } \\
\text { US finding }(\mathrm{N}=15), \mathrm{n}(\%)\end{array}$ \\
\hline $\begin{array}{l}\text { Abnormal findings } \\
\quad \text { Mass }\end{array}$ & $30(52.6)$ & $0(0)$ \\
Calcifications & $19(33.3)$ & $15(100)$ \\
$\quad$ Mass and calcifications & $8(14.1)$ & $0(0)$ \\
Dimensions & & $11(73.3)$ \\
$\leq 10 \mathrm{~mm}$ & $30(52.6)$ & $4(26.7)$ \\
$11-20 \mathrm{~mm}$ & $20(35.1)$ & $0(0)$ \\
$\geq 21 \mathrm{~mm}$ & $7(12.3)$ &
\end{tabular}

Lesions with and without a US correlate were considered separately.
Table 3. Characteristics of the lesions found on breast MRI

\begin{tabular}{lc}
\hline Breast MRI finding & Lesions, $\mathrm{n}(\%)$ \\
\hline Morphology & \\
$\quad$ Round & $15(36.6)$ \\
Oval & $21(51.2)$ \\
$\quad$ Irregular & $5(12.2)$ \\
Margins & \\
$\quad$ Well-defined & $30(73.2)$ \\
$\quad$ Irregular & $11(26.8)$ \\
Enhancement & \\
Intense (> 100\%) & $22(53.7)$ \\
Moderate (50-100\%) & $13(31.7)$ \\
Subtle (<50\%) & $6(14.6)$ \\
Early & $22(53.7)$ \\
Late & $19(46.3)$ \\
\hline MRI = Magnetic resonance imaging.
\end{tabular}

Table 4. Characteristics of the lesions upgraded during follow-up

\begin{tabular}{clll}
\hline Histology & Ultrasound & Mammography & $\begin{array}{l}\text { Risk factors / } \\
\text { symptoms }\end{array}$ \\
\hline $\begin{array}{c}\text { Phyllodes } \\
\text { tumor }\end{array}$ & $\begin{array}{l}\text { BI-RADS 3, lobulated, } \\
\text { hypoechoic, well- } \\
\text { defined margins }\end{array}$ & negative & palpable \\
Papilloma & $\begin{array}{l}\text { BI-RADS 3, round, } \\
\text { hypoechoic, well-de- } \\
\text { fined margins }\end{array}$ & negative & none \\
$\begin{array}{c}\text { Invasive } \\
\text { lobular } \\
\text { cancer }\end{array}$ & $\begin{array}{l}\text { BI-RADS 3, oval, } \\
\text { hypoechoic, well- } \\
\text { defined margins }\end{array}$ & negative & none \\
$\begin{array}{c}\text { Ductal } \\
\text { carcinoma } \\
\text { in situ }\end{array}$ & $\begin{array}{l}\text { BI-RADS 3, oval, } \\
\text { hypoechoic, well- } \\
\text { defined margins }\end{array}$ & negative & none \\
\hline
\end{tabular}

BI-RADS = Breast Imaging Report and Data System.

Overall follow-up ranged from 24 to 134 months (mean \pm SD, $63.3 \pm 15$ months). No interval changes were observed at shortterm follow-up. 4 lesions in 4 patients demonstrated modifications in their features, and the repetition of a histological evaluation was

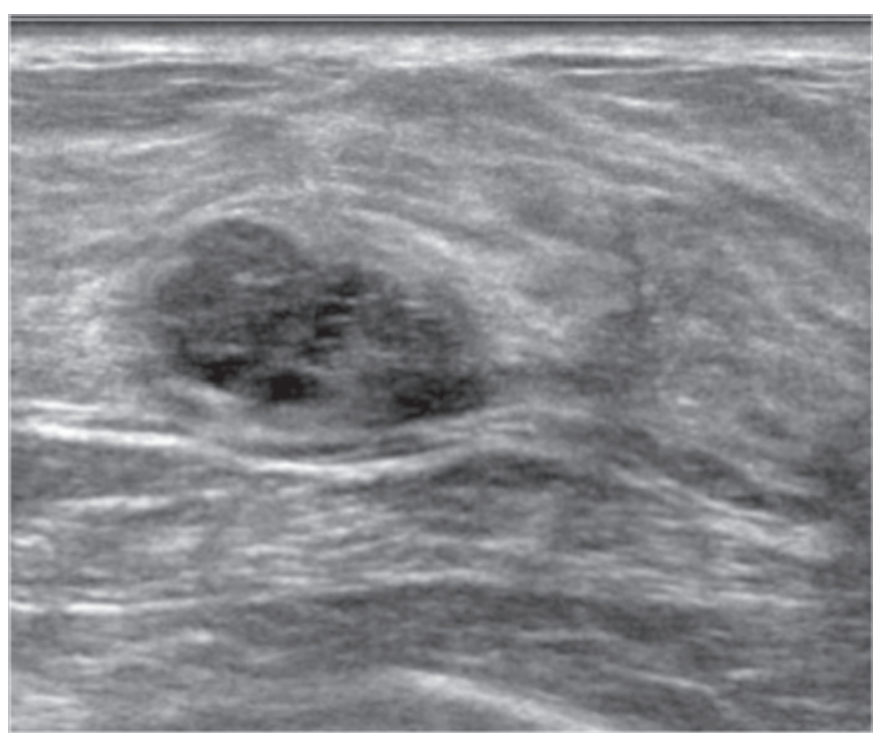

Fig. 1. A 26-year-old patient underwent US because of a palpable lesion. A lobulated lesion with well-defined margins, which was hypoechoic with anechoic areas, was found (BI-RADS 3). Biopsy was performed, in agreement with the patient, and histology showed a fibroadenoma. After 4 years, the lesion grew in size, from 17 to $24 \mathrm{~mm}$. The patient underwent a second biopsy, with histologic diagnosis of B3. A borderline phyllodes tumor was then diagnosed after surgical excision.

indicated (table 4). 2 lesions underwent a second CNB, and a surgical biopsy was performed in the other 2 cases. None of the patients underwent breast MRI.

The first of these 4 lesions was palpable at first diagnosis and showed an increase in size 4 years after biopsy; this was discussed with the patient, and the lesion was re-biopsied, with a diagnosis of B3. Surgical excision was performed and the diagnosis was a borderline phyllodes tumor (fig. 1). The second lesion demonstrated minimal growth after 1 year, which was associated with changes in the US features: increased hypoechogenicity and more indistinct margins. The lesion was re-classified as BI-RADS 4a; the biopsy was repeated, but was inconclusive (B1). The patient was sent to excisional biopsy, and histology showed a sclerosing papilloma with atypia (fig. 2). In the other 2 cases, only a slow increase in size was identified 3 years after biopsy: Surgical biopsy was performed and the diagnosis was invasive lobular carcinoma and 
Fig. 2. A 38-year-old patient underwent US. A small, round, hypoechoic lesion classified as BI-RADS U3 was found. In agreement with the patient, the lesion was biopsied and the result was a fibroadenoma. No changes were found at the 6-month re-scan. After 1 year, the lesion appeared round, more hypoechoic, with not very welldefined margins (a) and without significant vascularization (b), and it was re-classified as U4. Histology was repeated, but it was inconclusive (B1). The patient was sent to excisional biopsy and histology showed a sclerosing papilloma.
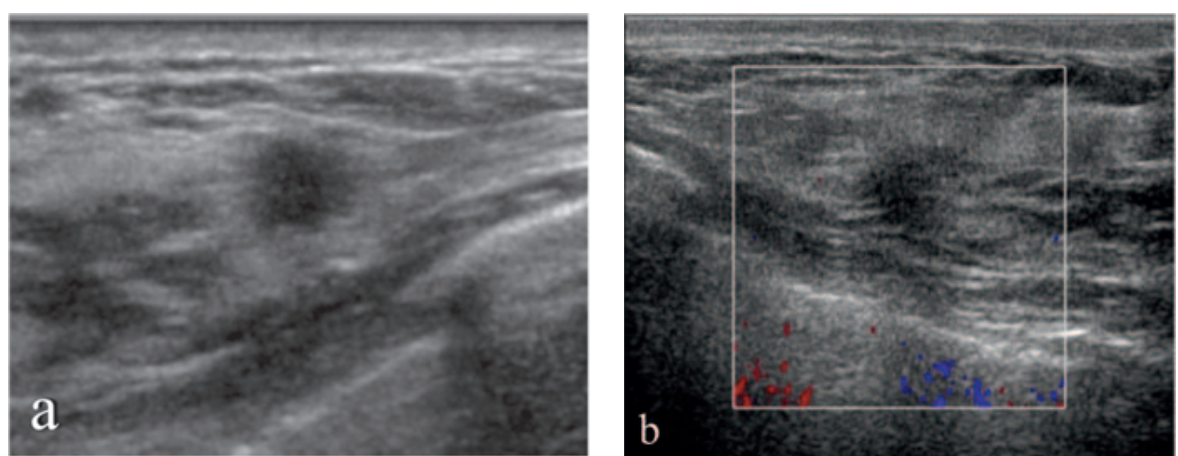

ductal carcinoma in situ, respectively. We considered these cases as FN at biopsy (4/349 lesions, 1.1\%). The overall NPV of biopsy was $98.9 \%$.

At follow-up, new benign lesions (classified as BI-RADS 2) were identified in 49 women $(49 / 318,15.4 \%)$. In 11 patients $(11 / 318$, $3.4 \%$ ), a new breast cancer was diagnosed on the same side or on the contralateral side in 6 and 5 cases, respectively. In another 6 women $(6 / 318,1.8 \%)$, high-risk lesions were identified, in 3 cases in the same breast, and in 3 cases in the contralateral breast of the known fibroadenoma.

\section{Discussion}

We performed a retrospective analysis on the follow-up of cases in which a lesion was found and initially classified as probably benign and in which histology revealed a fibroadenoma. We found that, during follow-up, none of the cases presented variations at 6 months after the biopsy. In $1.1 \%$ of the cases, the lesions were rebiopsied and a malignant or high-risk lesion was found. In all these cases, the changes in lesion features were identified at least 1 year after the first biopsy was obtained. Only $1(0.3 \%)$ of the 4 lesions was an invasive cancer.

The sensitivity of image-guided biopsy in distinguishing between benign and malignant lesions is very high [8-10]. Nevertheless, the risk of FN findings must be taken into consideration, as the percentage of FN results at US-guided CNB with a 14-G needle varies from 0 to $4.3 \%$ [11], and at VAB, with an 11-G needle with stereotactic guidance, from 0 to $3.3 \%$, in case of proven correct retrieval [12]. FN results may be ascribed to sampling errors, incomplete lesion sampling, and misjudgment in the presence of a discrepancy between histology and imaging evaluation. In some cases, the collection of a sufficient number of samples is hardly feasible, for example, because of substantial bleeding or difficulties in positioning and targeting [13]. Fibroadenoma usually presents with well-defined margins and a size greater than $5 \mathrm{~mm}$. As a consequence, the whole biopsy procedure is more often performed without difficulties and an adequate number of samples can be collected.

From a pathological point of view, the histological characterization of a fibroadenoma can be challenging, both because of overlapping lesion features and because of patient-related factors, such as young age and ongoing hormonal therapy. A possible misdiagnosis can occur between fibroadenoma and phyllodes tumor [14, 15]. Liberman et al. [16] reported that phyllodes tumors are usually characterized by a greater diameter and a lesion with a maximum diameter of more than $3 \mathrm{~cm}$ must be considered suspicious for a phyllodes tumor. In our study, the only phyllodes tumor found had a diameter of $2 \mathrm{~cm}$; thus, follow-up is essential to confirm the diagnosis, but, as the lesion is not particularly aggressive, a short-term follow-up is likely unnecessary.

Malignant tumor growth in a lesion already characterized as a fibroadenoma has been described [17-19], and is related to the epithelial component of the benign lesion. In our experience with CNB (about 10,000 cases), we found only 2 proven cases of cancer that had grown in a fibroadenoma, and which were not included in the study because of the lack of compliance with our inclusion criteria. Although it was not possible to verify this for the cancers included in our study, if we accept the hypothesis that these cases were also attributable to the growth of a malignant lesion inside the fibroadenoma, our data would show only $2 \mathrm{FN}$ cases (the phyllodes tumor and the papilloma), with an NPV of $99.7 \%$.

No significant changes in any of the high-risk and malignant lesions were detected at short-term follow-up. Leconte et al. [20] found an FN rate of $0.47 \%$ for fibroadenoma-like lesions in which a fine-needle aspiration biopsy (FNAB) was obtained under US guidance. These authors concluded that a short-term follow-up could be avoided when the cytology result was indicative of fibroadenoma and there was concordance between cytology and imaging. However, the study had a short follow-up period. Also, FNAB can be performed only by a pathologist with experience in cytology. Needle biopsy is not much more invasive compared to the FNAB, and allows a better evaluation of the specimens and a better characterization of the lesions.

Another recent study conducted by Manjoros et al. [21] analyzed a series of 200 benign lesions diagnosed at CNB in women with probably benign findings, and the results were similar to those of our series. In particular, malignancy was found in only 1 case $(0.5 \%)$, in a high-risk patient.

Short-term re-scanning after a histological diagnosis of fibroadenoma is common practice in many hospitals. We found that many patients did not show up at the short-term re-scan, or abandoned follow-up after 1 year (overall, 463/318; 59.3\%). The low FN rate of biopsies with a histologic diagnosis of fibroadenoma would allow a 
less stringent definition of the ideal timing for follow-up. This would be helpful to avoid the stress of a short-term recall, a circumstance that afflicts a high number of women, even when the precautionary approach is clearly explained to the patient.

Our study has some limitations: It is a retrospective study and data were collected in only 1 center highly specialized in breast imaging. Thus, many patients were lost to follow-up, and more studies are necessary to confirm our results. In addition, histological data were retrieved only by report and it was not possible to review specimens. A relatively high number of cancers were found. According to our retrospective data collection, only $5.6 \%$ of our population had a high risk of developing breast cancer due to personal or family history and none of them developed breast cancer in the biopsied lesion during the follow-up. The data were collected in a specialized breast center where many patients are sent after a recall from screening, after an abnormal examination in another facility, or due to clinical symptoms. Thus, it is likely that the patients included in the analysis had an overall risk higher as compared to the general population, despite not being at high risk for breast cancer.

Despite these limitations, we can conclude that a follow-up of 1 year is safe for breast lesions initially classified as BI-RADS 3 or $4 \mathrm{a}$ with a percutaneous histological diagnosis of fibroadenoma and that the short-term, 6-month follow-up can be avoided.

\section{Disclosure Statement}

The authors (P.C., M.B., M.M., V.L., C.Z.) declare no relationships with any companies, whose products or services may be related to the subject matter of the article. The authors state that this work has not received any funding.

\section{References}

1 Fornage BD, Lorigan JG, Andry E: Fibroadenoma of the breast: sonographic appearance. Radiology 1989; 172:671-675.

2 D’Orsi CJ, Sickles EA, Mendelson EB, Morris EA: ACR BI-RADS ${ }^{\circledR}$ Atlas, Breast Imaging Reporting and Data System, ed 5. Reston, American College of Radiology, 2013.

3 Graf O, Helbich TH, Fuchsjaeger MH, Hopf G, Morgun M, Graf C, et al.: Follow-up of palpable circumscribed noncalcified solid breast masses at mammography and US: can biopsy be averted? Radiology 2004;233:850-856.

4 Graf O, Helbich TH, Hopf G, Graf C, Sickles EA: Probably benign breast masses at US: is follow-up an acceptable alternative to biopsy? Radiology 2007;244: 87-93.

5 Harvey JA, Nicholson BT, Lorusso AP, Cohen MA, Bovbjerg VE: Short-term follow-up of palpable breast lesions with benign imaging features: evaluation of 375 lesions in 320 women. AJR Am J Roentgenol 2009;193: 1723-1730.

6 Ellis IO, Humphreys S, Michell M, Pinder SE, Wells CA, Zakhour HD: Best Practice No 179. Guidelines for breast needle core biopsy handling and reporting in breast screening assessment. J Clin Pathol 2004;57: 897-902.

7 Stavros AT, Thickman D, Rapp CL, Dennis MA Parker SH, Sisney GA: Solid breast nodules: use of sonography to distinguish between benign and malignant lesions. Radiology 1995;196:123-134.
8 Parker SH, Burbank F, Jackman RJ, Aucreman CJ, Cardenosa G, Cink TM, et al.: Percutaneous large-core breast biopsy: a multi-institutional study. Radiology 1994;193:359-364.

9 Lee J-M, Kaplan JB, Murray MP, Bartella L, Morris EA, Joo S, et al.: Imaging histologic discordance at MRIguided 9-gauge vacuum-assisted breast biopsy. AJR Am J Roentgenol 2007;189:852-859.

10 Di Loreto C, Puglisi F, Rimondi G, Zuiani C, Anania G, Mea Della V, Beltrami CA: Large core biopsy for diagnostic and prognostic evaluation of invasive breast carcinomas. Eur J Cancer 1996;32A:1693-1700.

11 Crystal P, Koretz M, Shcharynsky S, Makarov V, Strano S: Accuracy of sonographically guided 14-gauge core-needle biopsy: results of 715 consecutive breast biopsies with at least two-year follow-up of benign lesions. J Clin Ultrasound 2005;33:47-52.

12 Jackman RJ, Marzoni FA, Rosenberg J: False-negative diagnoses at stereotactic vacuum-assisted needle breast biopsy: long-term follow-up of 1,280 lesions and review of the literature. AJR Am J Roentgenol 2009;192: 341-351.

13 Youk JH, Kim E-K, Kim MJ, Lee JY, Oh KK: Missed breast cancers at US-guided core needle biopsy: how to reduce them. Radiographics 2007;27:79-94.

14 Tan PH, Ellis IO: Myoepithelial and epithelial-myoepithelial, mesenchymal and fibroepithelial breast lesions: updates from the WHO Classification of Tumours of the Breast 2012. J Clin Pathol 2013;66:465-470.
15 Gatta G, Iaselli F, Parlato V, Di Grezia G, Grassi R Rotondo A: Differential diagnosis between fibroadenoma, giant fibroadenoma and phyllodes tumour: sonographic features and core needle biopsy. Radiol Med 2011;116:905-918.

16 Liberman L, Bonaccio E, Hamele-Bena D, Abramson AF, Cohen MA, Dershaw DD: Benign and malignant phyllodes tumors: mammographic and sonographic findings. Radiology 1996;198:121-124.

17 Ooe A, Takahara S, Sumiyoshi K, Yamamoto H, Shiba E, Kawai J: Preoperative diagnosis of ductal carcinoma in situ arising within a mammary fibroadenoma: a case report. Jpn J Clin Oncol 2011;41:918-923.

18 Tajima S, Kanemaki Y, Kurihara Y, Okamoto K, Shimamoto $\mathrm{H}$, Okazaki $\mathrm{H}$, et al.: A case of a fibroadenoma coexisting with an invasive lobular carcinoma in the breast. Breast Cancer 2011;18:319-323.

19 Abe M, Miyata S, Nishimura S, Iijima K, Makita M, Akiyama F, et al.: Malignant transformation of breast fibroadenoma to malignant phyllodes tumor: longterm outcome of 36 malignant phyllodes tumors. Breast Cancer 2011;18:268-272.

20 Leconte I, Abraham C, Galant C, Sy M, Berlière M, Fellah L: Fibroadenoma: can fine needle aspiration biopsy avoid short term follow-up? Diagn Interv Imaging 2012;93:750-756.

21 Manjoros DT, Collett AE, Alberty-Oller JJ, Frazier TG, Barrio AV: The value of 6-month interval imaging after benign radiologic-pathologic concordant minimally invasive breast biopsy. Ann Surg Oncol 2013;20: 3163-3168. 